\title{
Advances in the delivery of buprenorphine for opioid dependence
}

This article was published in the following Dove Press journal:

Drug Design, Development and Therapy

28 August 2017

Number of times this article has been viewed

\section{Richard N Rosenthal' \\ Viral V Goradia ${ }^{2}$}

'Department of Psychiatry, Addiction Institute at Mount Sinai, Icahn School of Medicine at Mount Sinai, New York, ${ }^{2}$ Department of Psychiatry, Upstate Medical University, Syracuse, NY, USA
Correspondence: Richard N Rosenthal Icahn School of Medicine at Mount Sinai, Mount Sinai West, 1000 Tenth Avenue, 8C-02, New York, NY 10019, USA

Tel +l 2I 25236455

Fax + I 2125233477

Email richard.rosenthal@mountsinai.org
Abstract: Opioid use disorders (OUDs) have long been a global problem, but the prevalence rates have increased over 20 years to epidemic proportions in the US, with concomitant increases in morbidity and all-cause mortality, but especially opioid overdose. These increases are in part attributable to a several-fold expansion in the prescription of opioid pain medications over the same time period. Opioid detoxification and psychosocial treatments alone have each not yielded sufficient efficacy for OUD, but $\mu$-opioid receptor agonist, partial agonist, and antagonist medications have demonstrated the greatest overall benefit in OUD treatment. Buprenorphine, a $\mu$-opioid receptor partial agonist, has been used successfully on an international basis for several decades in sublingual tablet and film preparations for the treatment of OUD, but the nature of formulation, which is typically self-administered, renders it susceptible to nonadherence, diversion, and accidental exposure. This article reviews the clinical trial data for novel buprenorphine delivery systems in the form of subcutaneous depot injections, transdermal patches, and subdermal implants for the treatment of OUD and discusses both the clinical efficacy of longer-acting formulations through increasing consistent medication exposure and their potential utility in reducing diversion. These new delivery systems also offer new dosing opportunities for buprenorphine and strategies for dosing intervals in the treatment of OUD.

Keywords: opioid use disorder, buprenorphine, drug diversion, drug implants, depot medications, maintenance therapy, treatment adherence

\section{Introduction}

Opioid use disorders (OUDs) are a continuing and increasing worldwide problem ${ }^{1}$ that, in the US, have become epidemic over the last two decades, led by nonmedical use of prescription pain medications. ${ }^{2}$ Compared to those without OUD, patients with OUD have a significantly higher disease burden, ${ }^{3}$ including especially high rates of mortality for those not treated within specialty addiction treatment settings. ${ }^{4}$ In addition to the medical, economic, and social impairments typically associated with OUD, fatal overdoses related to opioids were at their highest in US history in 2015, including 12,727 deaths due to natural or semisynthetic opioids, 12,989 deaths due to heroin, and excluding methadone, a $72.2 \%$ increase over 2014 in death rate due to synthetic opioids such as fentanyl. ${ }^{2,5}$ Thus, the need for effective treatment is emergent, and medically supervised agents with partial- or full-agonist properties such as buprenorphine or methadone are recommended as first-line maintenance interventions, supported by high-quality evidence. ${ }^{6}$ However, less than half of the $\sim 2.5$ million persons in the US with an OUD have any access to evidence-based pharmacological treatment for it. ${ }^{7}$ In contrast, most treatment provided in the US for substance use disorders (SUDs) is psychosocial, and while there is a significant evidence base in support of psychosocial treatment of alcohol- and stimulant-based SUD, the evidence is 
not robust in support of only psychosocial treatment of OUD provided after detoxification from opioids. ${ }^{8,9}$ More specifically, maintenance with buprenorphine has higher efficacy on treatment retention and adverse events than detoxification or psychological treatment. ${ }^{10}$ Among Medicaid recipients, OUD patients treated with methadone or buprenorphine have significantly lower relapse rates as well as lower overall costs of treatment. ${ }^{11}$ In addition, psychosocial interventions do not appear to add additional efficacy to effective medical maintenance treatments for OUD generally. ${ }^{12}$ Therefore, given the high rates of OUD related to the use of prescription pain medications compared to other sources of opioids in the US, it is imperative that more evidence-based medical treatments are made available to those with OUD. This should not only address those with current OUD but may also provide secondary prevention, as problematic use of prescription pain medications is a predominant pathway to the use of heroin and has played a significant role in the current heroin epidemic in the US. ${ }^{13}$ However, it is also important to note that diversion of prescribed medications is not localized solely to medications with abuse liability or to those recipients with OUD. ${ }^{14}$

\section{Diversion of prescribed opioids}

The increased prescribing of opioid pain medications has supported a concomitant and dramatic increase in illicit use, diversion, and overdose.,15 Patterns of high-intensity prescribing of opioid pain medications by physicians in hospital emergency departments significantly predict patients' longterm opioid use. ${ }^{16}$ The risk for long-term opioid use increases with initial prescriptions for long-acting opioids, initial prescription for $>10$ or $>30$ days, $>2$ prescriptions, and a cumulative dose of $700 \mathrm{mg}$ morphine equivalents. ${ }^{17}$ One-toone educational visits support prescribing lower quantities of prescription analgesics for acute pain and reduce rates of prescribing of high-dose prescription pain medications. ${ }^{18} \mathrm{In}$ addition, improving controls over prescribing through legal and regulatory constraints in pain clinics and mandatory clinician review of prescription drug monitoring program data has impact on the rates of prescription opioid overdose deaths, but these interventions alone appear insufficient to address the epidemic. ${ }^{19}$ Another opportunity to impact the diversion and illicit the use of prescription medications, whether prescribed for treatment of pain or for maintenance treatment of OUD, is to alter the drug delivery system. The need for better delivery systems clearly applies to the use of opioid pain medications - in one study, four fifths of patients entering treatment for OUD were documented as having accelerated the delivery of prescription pain tablets by chewing, insufflating, or injecting it intravenously. ${ }^{20} \mathrm{In}$ the US, immediate release prescription opioids are prescribed at much higher rates than long-acting formulations and have associated higher rates of abuse and diversion. ${ }^{21}$ In addition, diversion of opioid maintenance medications by patients participating in opioid substitution treatment programs is also substantial. For example, a study in Sweden including a $40.8 \%$ sample across 11 opioid treatment programs found that $67.6 \%$ of patients interviewed had ever diverted their maintenance medications, mostly by giving it away or selling it while participating in the program, and $24.1 \%$ had done so in the past month, with significantly higher diversion risks among younger patients. ${ }^{22}$ Addressing buprenorphine specifically, data from Australian opioid treatment programs and pharmacies suggest that among patients with OUD, the 1-year prevalence of self-reported buprenorphine diversion is $15 \%-24 \%{ }^{23,24}$ People with OUD frequently use diverted buprenorphine to medicate opioid withdrawal because of their difficulty in accessing treatment..$^{25}$

\section{Impact of medication delivery systems on diversion}

Delivery systems for OUD treatment medications with opioid agonist activity seem to make a difference in diversion. From 2003 to 2009, buprenorphine/naloxone tablets were sold at an increasing rate of an additional 34 million tablets per year for the purposes of OUD treatment in the US, with a parallel increase in diversion. ${ }^{26}$ In Australia, surveys of injection drug users, patients receiving opioid substitution medication through offices, clinics, and pharmacies, and clinicians prescribing opioid substitution treatment revealed that the sublingual combination buprenorphine/naloxone tablet formulation was injected at a lower frequency than the buprenorphine mono product, validating the hypothesized deterrence of the combination tablet form, ${ }^{27}$ but that overall both the buprenorphine formulations were diverted at a higher rate than methadone dispensed as a liquid. ${ }^{28}$ However, novel formulations of sublingual buprenorphine preparations can differentially impact nonadherence and diversion rates. A double-blind double-dummy study comparing buprenorphine/naloxone in tablet and film formulations among patients already receiving treatment for OUD, while finding no between-group differences in efficacy measures, adverse, events, or pharmacokinetics, demonstrated that the films dissolved faster, suggesting that it would be less susceptible to diversion after administration. ${ }^{29}$ In addition, US data from poison control centers, drug diversion police investigations, patients attending opioid treatment programs, and college student surveys found that the combination 
buprenorphine film is diverted or intentionally abused significantly less frequently in all the settings than the combined tablet formulation, suggesting that a different delivery system may be involved. ${ }^{30}$ However, while the Australian data found that a lower proportion of patients in opioid substitution treatment prescribed buprenorphine/naloxone films being injected than that for buprenorphine/naloxone tablets or the mono tablet product, the films were diverted and used at similar rates to the combination tablet products by injecting drug users who were not in treatment. ${ }^{31}$ Thus, improvements in the delivery of sublingual buprenorphine have supported reductions in diversion, but there may be considerable opportunity to develop other delivery strategies that might further reduce diversion and rates of illicit use.

When patients with OUD receive medications with opioid agonist activity such as methadone or buprenorphine for maintenance treatment, it is reasonable to expect that a delivery system that reduces the risks of altered self-administration or diversion is also likely to increase its effectiveness for clinical stabilization through increased treatment exposure.

\section{Buprenorphine pharmacology}

Buprenorphine is a partial agonist with high affinity for the $\mu$-opioid receptor but weak agonist activity compared to full agonists such as methadone and demonstrates high affinity for and antagonism at the $\kappa$ - and $\delta$-opioid receptors. ${ }^{32}$ Owing to its partial agonist properties at $\mu$-opioid receptor, buprenorphine will precipitate opioid withdrawal in individuals who are physically dependent on full opioid agonists such as morphine, but compared to pure $\mu$-agonists is also relatively safer at high doses when administered alone, owing to its inverted u-shaped dose-response curve for respiratory depression demonstrated in animal models and its plateauing at increasing doses as evidenced in human adults. ${ }^{33,34}$ Sublingual buprenorphine taken for the treatment of OUD has a relatively long duration of action with a $\mathrm{T}^{1 / 2}$ of $24-60$ hours. ${ }^{33}$ At the nociceptin/orphanin FQ (N/OFQ) receptor (formerly opioid receptor-like 1), buprenorphine acts as a low-affinity, weak partial agonist in humans, where its action opposes the analgesic and rewarding properties of morphine. ${ }^{32}$ In animal models, buprenorphine also acts as an antagonist of betaendorphin-mediated G-protein activation at the purported $\varepsilon$-opioid receptor and binds it with high affinity, blocking $\beta$-endorphin-induced antinociception. ${ }^{35,36}$

\section{Efficacy trials of buprenorphine for OUDs}

A 2014 Cochrane review evaluated maintenance treatment with buprenorphine compared to placebo and to methadone maintenance in the management of opioid dependence. ${ }^{37}$ The trials included different formulations of buprenorphine: sublingual solution, sublingual tablets, combined buprenorphine/ naloxone sublingual tablet, and an implant. The review included 31 trials $(5,430$ participants), with the evidence graded at moderate to high quality. Compared with placebo, high-dose buprenorphine (16 mg) can reduce illicit opioid use effectively. There is a moderate quality of evidence that high-dose buprenorphine ( $\geq 16 \mathrm{mg}$ ) was more effective than placebo in suppressing illicit opioid use measured by urinalysis in the trials; however, in two of the studies from the meta-analysis, low-dose (2-6 mg) and medium-dose (7-15 mg) buprenorphine did not suppress illicit opioid use measured by urinalysis better than placebo. ${ }^{38,39}$ Regarding retention of participants in the treatment, buprenorphine at all doses examined was superior to placebo medication. At fixed doses above $7 \mathrm{mg}$ per day, buprenorphine was comparable for treatment retention to fixed doses at $40-85 \mathrm{mg}$ of methadone per day. However, buprenorphine retains patients in the treatment less effectively than methadone if prescribed in a flexible dose regimen according to patient need or at a low, fixed dose ranging from 2 to $6 \mathrm{mg}$ per day. ${ }^{37}$ There were no study findings of differences in decreased use of other abused substances such as cocaine or benzodiazepines, criminal activity, or mortality. ${ }^{37}$

\section{Route of administration may affect OUD treatment outcome}

Transmucosal medications administered orally for OUDs, in addition to adherence issues specifically related to the route of administration (taste, dosing schedule), may generate adherence problems related to the pharmacological properties of the medication or its side effects, such as low adherence seen with orally administered naltrexone. ${ }^{40}$ However, even when adherence is controlled with observed dosing, medications for OUDs may demonstrate differential efficacy by the route of administration. For example, a double-blind double-dummy 24-week study of observed dose of oral naltrexone, sublingual buprenorphine, and placebo was conducted in Malaysia with 126 recently detoxified heroindependent patients which provided manual-guided weekly individual and group drug counseling and demonstrated consistent, significant superiority findings for sublingual buprenorphine ( $8 \mathrm{mg} /$ day mean dose) compared to oral naltrexone (50 mg/day mean dose) and placebo in days to both first and sustained heroin use as well as in retention in treatment. ${ }^{41}$ Similarly, a retrospective case-control study of medical charts of patients $(n=248)$ treated in India for opioid addiction showed that the odds of retention in treatment 
for patients maintained on buprenorphine is $4.5(P<0.005)$ times greater than patients maintained on oral naltrexone and 7 times $(P<0.001)$ greater than patients receiving only psychosocial intervention. ${ }^{42}$ A 12 -week, double-blind randomized clinical trial at a single clinical site in Iran also compared the effectiveness of thrice-weekly dosing of oral naltrexone and sublingual buprenorphine/naloxone for OUD. The findings demonstrated that sublingual buprenorphine/naloxone was associated with a significantly greater mean number of opioid-negative urine tests as well as better treatment retention than oral naltrexone, but neither significantly greater duration of time to relapse to illicit opioid use nor proportions with 12 -week abstinence, which ranged from $8 \%$ in the naltrexone group to $16 \%$ in the buprenorphine group. ${ }^{43}$ Thus, although sublingual buprenorphine appears overall to be more efficacious than oral naltrexone, buprenorphine results suggest that a large percentage of those treated on orally or sublingually administered medications may be at risk for continued illicit opioid use, even when adherent to treatment. After each sublingual dose of buprenorphine, plasma concentrations peak within several hours and drop steeply with associated subjective symptoms of withdrawal in a substantial subpopulation..$^{44}$ In addition, sublingual formulations of buprenorphine produce variable buprenorphine blood levels and require frequent dosing that potentially jeopardizes medication adherence. ${ }^{45}$ Systematic reviews suggest that, overall, the full benefits of medical treatments for chronic medical conditions are not obtained as most approaches intended to improve patient adherence to medication are complex and not so effective. ${ }^{46}$ Reducing poor medication adherence by extending the dosing cycle is a more specific intervention that may improve treatment outcomes. ${ }^{47} \mathrm{~A}$ longer-acting formulation of buprenorphine might address the diurnal variation that has been described and observed with daily dosing and thus reduce vulnerability to relapse.

\section{Public health impact of poor adherence}

An important consequence related to poor medication adherence is the public health impact of drug exposure of prescribed maintenance opioid substitution agonist and partial agonist in those other than the intended recipient, namely, exposure through intentional diversion and illicit administration or through accidental use. Systematic review of the literature from the European Union suggests that diversion of both methadone and buprenorphine is substantial, which implies that a relevant proportion of those being prescribed as medications are not adherent to treatment. ${ }^{48}$ In addition, a less frequent but significant public health risk is the unintentional diversion to minors due to poor control over the medication supply, resulting at times in an accidental pediatric intoxication or overdose. ${ }^{49,50}$ Buprenorphine was reported by the US Centers for Disease Control and Prevention for 2010-2011 to have caused the largest percentage of emergency hospitalizations in children under 6 years of age compared to any other medication. ${ }^{51}$ Widespread implementation of unit-dose films in child-resistant packaging probably contributed to a decrease in buprenorphine-related pediatric emergency room visits from 2013 to 2015, but the introduction of generic buprenorphine tablets in multi-dose bottles may contribute to a leveling off of the reductions in pediatric poisoning rates or even an increase. ${ }^{52}$

\section{New medication delivery approaches for treating OUD}

The adherence problem with conventional oral daily dosing of methadone and buprenorphine maintenance and naltrexone has led to the search for a treatment that maximizes both efficacy and adherence and, in the case of medications with abuse liability, reduces the risk of diversion.

One strategy that can potentially affect both clinical outcomes and reduce diversion is to alter the dosing frequency of medications. For example, Levo-alpha-acetyl-methadol (LAAM) provided an alternative to the daily dosing with methadone with twice or thrice weekly dosing. In studies comparing LAAM to methadone, LAAM consistently demonstrated non-inferiority and trended toward improved treatment retention and lower opioid use. ${ }^{53-55}$ In addition, it appeared to be better at stabilizing withdrawal symptoms, perhaps due to the low fluctuation in active plasma metabolites over the dosing interval. ${ }^{56}$ However, following reports of severe cardiac-related adverse events, including QT interval prolongation, Torsades de Pointes, and cardiac arrest, LAAM was discontinued from the European market in 2001 and the US market in 2004. ${ }^{57}$

Although beyond this paper's focus on buprenorphine, it is noteworthy to mention the $\mu$-opioid receptor antagonist naltrexone, since long-acting formulations can attend to issues of treatment adherence in a population that has been well described as poorly adherent to daily oral administration of opioid receptor antagonists for OUDs. ${ }^{40,58-60}$ In general, long-acting naltrexone preparations reduce illicit opioid use and positively impact morbidity and mortality. ${ }^{61}$ In 2010, the US Food and Drug Administration approved a long-acting injectable intramuscular form of naltrexone to treat DSM IV Opioid Dependence. Randomized controlled and open trials have demonstrated the efficacy of monthly long-acting 
injectable naltrexone as compared to daily oral formulations or placebo in the treatment of opioid dependence, suggesting that a long-acting injectable naltrexone preparation sustains exposure to treatment and improves outcomes. ${ }^{62-65}$ Similarly, implantable forms of naltrexone demonstrate advantages over the daily oral formulation in maintaining effective blood levels and in relapse to heroin use. ${ }^{66,67}$

\section{Transmucosal buprenorphine}

All buprenorphine sublingual or buccal formulations can be diverted for illegal sale and used illicitly. In situations where medications for OUD are being administered, such as in opioid treatment programs, changes to the formulation may impact the patient's ability to subvert the administration process and divert the medication. A recent (2014) addition to the transmucosal buprenorphine armamentarium for OUD is the development of BioErodible MucoAdhesive technology buccal buprenorphine/naloxone film (Bunavail, BioDelivery Sciences International, Inc, Raleigh, NC, USA) that adheres to the inside of the cheek while dissolving. ${ }^{68}$ The bilayer film has a backing layer facing the oral cavity that reduces oral administration of buprenorphine while the drug-containing layer has direct mucosal contact, which increases bioavailability compared to other transmucosal buprenorphine film preparations. ${ }^{69}$ While an open-label 12-week conversion study among participants $(\mathrm{N}=249)$ previously stabilized for at least 30 days on sublingual buprenorphine-naloxone tablets or film for OUD demonstrated that switching to a bioequivalent dose of buprenorphine in the buprenorphine-naloxone buccal film was well tolerated, efficacious, and safe, there does not appear to be any advantage over other transmucosal formulations with respect to diversion except in situations where the medicine is administered rather than prescribed or dispensed, as the buccal film, once applied, adheres strongly to the oral mucosa and dissolves completely. ${ }^{69}$

However, a large current component of medication treatment for OUD in the US is through prescription by physicians in office-based practice. As such, changes to the transmucosal buprenorphine delivery systems for selfadministered doses are unlikely to significantly impact the risk for improper or accidental use or diversion. Thus, a reasonable strategy to further address and reduce diversion is to develop long-acting preparations that are administered by a treating clinician and are less under the control of the patient. Long-acting preparations of buprenorphine have been tested for administration using 3 different delivery systems depot injections, transdermal patches, and subdermal implants. Part of the efficacy of transmucosally delivered buprenorphine is its pharmacological activity in reducing the reinforcing effects of co-administered opioid agonists, and this should thus be a characteristic of all long-acting preparations whether agonist, partial agonist, or antagonist, regardless of delivery system.

\section{Buprenorphine depot preparations}

Several depot forms of buprenorphine have been developed for subcutaneous administration. One formulation (Norvex; Biotek, Inc., Woburn, MA, USA), currently unavailable, used a biodegradable polymer 100-150 micron microcapsule depot technology consisting of buprenorphine base and a poly lactide-coglycolide polymer. ${ }^{70}$ After being administered as a subcutaneous injection, the plasma buprenorphine level increases to peak on day 2 or 3 and then decreases gradually to zero over 6 weeks. ${ }^{71}$ A small 6-week double-blind randomized controlled trial $(\mathrm{N}=15)$ of subcutaneous injection of $58 \mathrm{mg}$ buprenorphine depot versus depot placebo in opioid-dependent participants demonstrated that the depot medication was effective in suppressing symptoms of withdrawal and provided significant blockade of opioid receptors as evidenced by reduced objective and subjective responses to hydromorphone challenges. ${ }^{70}$

Another buprenorphine preparation (RBP-6000; Indivior, Richmond, VA, USA) that has been developed for monthly administration contains $200 \mathrm{mg} / \mathrm{mL}$ of buprenorphine base in a precipitation delivery system of biodegradable polylactideco-glycolide polymer and biocompatible solvent (N-methylpyrrolidone), which in contact with water, solidifies at the surface in the subcutaneous space ${ }^{72}$ and provides sustained release of buprenorphine over a minimum of 28 days through diffusion and polymer degradation. ${ }^{73}$ A $300 \mathrm{mg}$ dose of depot buprenorphine (RBP-6000) injected subcutaneously into the abdomen of subjects previously inducted to 8-24 mg of sublingual buprenorphine/naloxone daily reduced the mean "drug liking" visual analog scale scores to close to that of placebo after intramuscular 18 and $6 \mathrm{mg}$ hydromorphone challenges. A US study demonstrated that a $300 \mathrm{mg}$ dose of RBP-6000 provided a potent blockade of the subjective effects and reinforcing efficacy of hydromorphone in subjects with moderate or severe OUD. ${ }^{74}$ In addition, pharmacokinetic analysis of RBP-6000 showed that a subcutaneous dose of $300 \mathrm{mg}$ every 28 days rapidly achieved effective drug exposure after the initial injection and maintained effective levels during chronic treatment. A simulated 2-week drug holiday demonstrated $\mu$-opioid receptor occupancy consistently above $80 \%$ without loss of drug efficacy. ${ }^{75}$ A double-blind randomized controlled 24 -week study was conducted at 
38 locations in the US among treatment-seeking participants with moderate or severe OUD who were first inducted and dose adjusted to sublingual buprenorphine film at 8-24 mg over a 7-14 day period. Once assessed as stable with respect to objective and subjective withdrawal, participants were randomized and then transitioned to $300 \mathrm{mg}$ of subcutaneous buprenorphine for 2 monthly injections followed by low-dose (100 mg) buprenorphine for 4 injections, or 6 monthly high-dose buprenorphine (300 mg) injections, or 6 equivolume subcutaneous monthly placebo injections. Injections were given each month on alternate sides of the participant's abdomen (https://ClinicalTrials.gov/ct2/ show/NCT02357901). The between-groups cumulative distribution function of the percentage of opioid-negative urine samples plus self-reported non-use of opioids during 2-6 months of the study was significant $(P<0.0001)$ for both active treatment groups compared to the placebo condition. In addition, a between-groups comparison of responders defined as those with at least $80 \%$ of urine samples being negative for illicit opioids plus self-reports being negative for illicit opioid use over 2-6 months of the study was also significant $(P<0.0001)$ for each of the active treatment groups against placebo. $^{76}$

A depot buprenorphine preparation that uses a different delivery strategy from poly lactide-coglycolide microcapsules is based on the characteristics of certain low-viscosity lipids in contact with aqueous media to self-assemble into reversed-phase "water-in-oil" nonlamellar liquid crystal nanoparticle gels. ${ }^{77,78}$ The preparation CAM2038 uses this technology (FluidCrystal ${ }^{\circledR}$; Camurus AB, Lund, Sweden) to deliver buprenorphine in a low-viscosity two-lipid medium that can be delivered through a small 23-gauge needle as a premixed weekly or monthly subcutaneous injection. After injection, the depot, in contact with interstitial aqueous fluid, transforms into a viscous liquid-crystal gel phase that elutes buprenorphine at a predictable rate as the depot is biodegraded. Two dosage strengths have been developed, one for weekly injection $(50 \mathrm{mg} / \mathrm{mL})$ and one for monthly injection (356 mg/mL). An international open-label multisite safety/ efficacy study of CAM2038 is in progress to test 1-year safety in $\mathrm{N}=100$ outpatients with OUD who are currently maintained on sublingual buprenorphine or who are seeking treatment (https://ClinicalTrials.gov/ct2/show/NCT02672111). Subjects were given weekly subcutaneous buprenorphine injections of $0.16,0.32,0.48$, or $0.64 \mathrm{~mL}$, corresponding to doses of $8,16,24$, and $32 \mathrm{mg}$, respectively, to either replace their current sublingual buprenorphine dose or initiate maintenance treatment with clinical titration and then were maintained on the weekly dose or transitioned to monthly $0.18,0.27,0.36$, or $0.45 \mathrm{~mL}$ subcutaneous injections corresponding to doses of $64,96,128$, or $160 \mathrm{mg}$, respectively. A Phase III randomized, double-blind, active-controlled, parallel group, multicenter 24-week non-inferiority trial in $\mathrm{N}=428$ participants with moderate to severe DSM-5 OUD tested CAM2038 against sublingual buprenorphine in 36 US sites, where a responder was defined as $\geq 33 \%$ (4 of 12) Phase I urine toxicology tests and 67\% (4 of 6) of Phase II urine toxicology tests being negative for illicit opioids and illicit opioid use by self-report (https://ClinicalTrials.gov/ ct2/show/NCT02651584). At randomization, the participants were induced to daily sublingual buprenorphine with weekly placebo SC injections or weekly SC CAM2038 injections with daily placebo SL tablets. During the 12-week Phase II study, subjects in the SL Bup group continued treatment with monthly dispensing of daily SL BPN treatment and began monthly placebo SC injections, whereas the group receiving weekly CAM2038 began monthly injections and were dispensed daily SL placebo at monthly visits. The study demonstrated SC buprenorphine preparation was non-inferior to sublingual buprenorphine in the responder rate $(\mathrm{CI}-3.5 \%$, $10.4 \% ; P<0.001)$ and percentage of urine samples negative for illicit opioids ( $\mathrm{CI}-0.2 \%, 13.7 \% ; P<0.001)$. The study was also designed to test the superiority of the combined percent of urine samples negative for illicit opioids and/ or self-reported illicit opioid use over weeks 5-24, and it demonstrated significant results $(P=0.004)$ in favor of the subcutaneous injection group. ${ }^{79}$

\section{Transdermal buprenorphine}

A transdermal buprenorphine (46.6 mg) patch has also been investigated for the treatment of opioid dependence. An open-label 10-day detoxification trial in opioid-dependent participants $(\mathrm{N}=9)$ on a research unit found that while applied for 3 days, the patch was safe and well-tolerated, delivered a mean of $1.9 \mathrm{mg} /$ day of buprenorphine with a peak average plasma concentration of $60 \mathrm{ng} / \mathrm{mL}$ at 48 hours, continued the suppression of opioid withdrawal as evidenced by normalized physiologic measures, and subjective withdrawal symptoms reduced by about $50 \%{ }^{80}$ As an analysis of used patches in the study revealed that there was a substantial reservoir of buprenorphine remaining after the initial use, a replication open-label study extended the patch application to 7 days of the 10-day detoxification protocol for $(\mathrm{N}=12)$ opioid-dependent volunteers pre-stabilized with hydromorphone to suppress withdrawal symptoms. ${ }^{81}$ A significant suppression of withdrawal was maintained during the week 
of patch application and during the 24 hours after the patch was removed, consistent with buprenorphine plasma levels peaking at 48 hours after the patch was applied and sustaining mean levels $>0.5 \mathrm{ng} / \mathrm{mL}$ for 96 hours with a steady decline thereafter. This formulation was safe and, with the prior results, suggested clinical utility for opioid detoxification. ${ }^{81}$ Interestingly, using Researched Abuse, Diversion and Addiction-Related Surveillance (RADARS) System Poison Center data, a different buprenorphine transdermal preparation for the treatment of chronic pain was compared to other buprenorphine products, fentanyl patches, extended release opioid formulations, and extended release tramadol, which demonstrated the lowest rates of abuse and diversion. ${ }^{82}$

\section{Buprenorphine implant}

A subdermal formulation containing $90 \mathrm{mg}$ of buprenorphine blended homogeneously with a biocompatible nonbiodegradable form of ethylene vinyl acetate polymer and extruded into a $26 \times 2.5 \mathrm{~mm}$ rod-shaped implant was developed and approved for use by the US FDA in May 2016 (Probuphine; Braeburn Pharmaceuticals, Princeton, NJ, USA). In animal studies, buprenorphine release was dependent on the implant surface area, which determined the rate of dissolution and passive diffusion through the polymer matrix. ${ }^{45}$ In an initial open-label dose-finding safety/efficacy trial of the buprenorphine implants, post-implantation pharmacokinetic analysis in $\mathrm{n}=12$ heroin-dependent subjects demonstrated an initial pulse release with a peak at about 16 hours when 4 implants were administered, followed by a slow decrease to steady state by 21 days, and then a relatively constant linear release of buprenorphine from the implants until implant removal at 6 months. ${ }^{83}$ These pharmacokinetic data demonstrated an absence of plasma peaks and troughs typically demonstrated with daily dosing of medications. After explantation, the mean terminal half-life of plasma buprenorphine was about 24 hours, in similar range to that of sublingual and buccal buprenorphine. ${ }^{83-85}$

Recent Phase III, multisite placebo-controlled studies support the use of long-acting buprenorphine $80 \mathrm{mg}$ implants in the treatment of OUD. Two 24-week, randomized, double-blinded placebo-controlled multisite trials have been conducted in the US among participants with DSM-IV opioid dependence first stabilized clinically on $12-16 \mathrm{mg}$ of sublingual buprenorphine and provided standardized individual drug counseling. The first study randomized 162 participants at a 2:1 ratio to receive 4 buprenorphine $(80 \mathrm{mg})$ implants $(n=108)$ or 4 placebo implants $(n=55)$. Those randomized to buprenorphine implants had more than double the study completion rate compared to those who received placebo implants $(65.7 \%$ vs $30.9 \%)(P<0.001)$. The buprenorphine implant group also demonstrated significantly more urine samples negative for illicit opioids during weeks 1 through $16(P=0.04)$, and a mean percentage of urine samples that tested negative for illicit opioids across weeks 1 through 16 of $40.4 \%$ versus $20.8 \%$ in the placebo group ${ }^{86}$ The buprenorphine implant group participants also had significantly fewer clinician-rated withdrawal symptoms, lower opioid dependence severity and greater global improvement, and lower patient-rated symptoms of withdrawal and craving compared to the placebo implant recipients. ${ }^{86}$ The second study was also a double-blind, placebo-controlled trial, which evaluated the safety and efficacy of four $80 \mathrm{mg}$ buprenorphine implants $(n=114)$ versus placebo implants $(n=54)(2: 1$ randomization), as well as conducting a non-inferiority comparison of buprenorphine implants to open-label sublingual buprenorphine/naloxone tablets (12-16 mg/day, continued after study induction; $n=119$ ) for the treatment of opioid dependence. ${ }^{87}$ In this case, the main outcome measure was the percentage of negative urine samples for opioids collected from weeks 1 to 24, examined as a cumulative distribution function, and for which the buprenorphine implant group was significantly different from placebo $(P<0.0001)$. As in the prior study, ${ }^{86}$ active implant subjects had a higher study completion rate relative to placebo $(64 \%$ vs $26 \%, P<0.0001)$, lower clinician-rated withdrawal and higher global improvement ratings, lower subjects' ratings of withdrawal and craving, and better subjects' ratings of global improvement. Regarding the comparison with continued open-label sublingual buprenorphine/naloxone, the buprenorphine implants were non-inferior on the percentage of urine samples negative for opioids over the 24 weeks of study. ${ }^{87}$

In both the studies, the most frequent adverse events were within the class of implant site reactions; however, the rates in the first study were $52.7 \%$ in the placebo group and $56.5 \%$ in the buprenorphine group ${ }^{86}$ whereas the rates in the second study were $25.9 \%$ in the placebo group and $27.2 \%$ in the buprenorphine group. ${ }^{87}$ The differences in adverse events between the Ling et $\mathrm{al}^{86}$ and Rosenthal et $\mathrm{al}^{87}$ studies are likely due to improvements in the implantation methodology in the second study, which instituted applying a pressure bandage for 24 hours immediately post implantation and closure, and also replaced the original dull-ended insertion applicator with a specially designed sharp-edged beveled insertion applicator, since the original applicator was associated with more tissue adhesions to the implants that resulted in more implant fractures on explantation. ${ }^{87}$ 


\section{Implants in clinically stable populations}

Different from that of office-based treatment of OUDs with buprenorphine, the first two multisite clinical trials of buprenorphine implants were conducted in a treatment center setting with patients new to pharmacotherapy for opioid dependence, so as to optimize patient safety through intensive data assessments and capacity to rapidly modify the treatment. ${ }^{88}$ However, issues raised in the literature suggested that it was important to compare the implants directly to sublingual buprenorphine and to use a metric such as number needed to treat (NNT) or effect size in order to establish the clinical significance, ${ }^{89,90}$ although the median urine samples negative for illicit opioids of $40.7 \%$ in the buprenorphine implant group and $20.8 \%$ in the placebo group was considered to be a clinically meaningful difference in patients new to treatment. ${ }^{86,91}$ Having demonstrated safety and efficacy against placebo, the third randomized trial of buprenorphine implants (PRO-814) recruited patients on stable doses of buprenorphine who were receiving treatment in office-based practices. A double-blind, double-dummy, randomized controlled noninferiority study was conducted among 177 patients who were treated for at least 6 months with sublingual buprenorphine and were deemed stable by their treating clinician on a dose $\leq 8 \mathrm{mg}$ for at least 90 days with no evidence of illicit opioid use. ${ }^{92}$ Participants who were randomized to either 4 buprenorphine implants ( $80 \mathrm{mg}$ each) and placebo sublingual tablets or 4 dummy implants and buprenorphine sublingual tablets at their pre-randomization dose were considered responders if they demonstrated abstinence from illicit opioids by urine testing or self-report for at least 4 of the 6 study months. The proportion of responders was $87.6 \%$ with sublingual buprenorphine and $96.4 \%$ with buprenorphine implants, meeting a predefined noninferiority threshold $(P<0.001)$. Once the non-inferiority threshold was met, chi-square analysis of the $8.8 \%$ difference in responder rates demonstrated superiority of the buprenorphine implant ( $P=0.03$ ). In addition, significantly more of the implant group (85.7\%) compared to the sublingual group (71.9\%) were abstinent of illicit opioids over all 6 months of the study $(P<0.03$; NNT $=7.25$ against sublingual buprenorphine as the base intervention), and time to first use of illicit opioids was longer in the active implant group $(P=0.04) .{ }^{92}$ Taken together, these findings suggest that buprenorphine implants may be superior to the sublingual route of administration for the maintenance of long-term abstinence from illicit opioids. In all the three multisite randomized controlled studies of buprenorphine implants, but especially in the last where subjects were already clinically stable at lower doses
( $\leq 8 \mathrm{mg}$ ), conversion from sublingual buprenorphine to the implant was clinically non-disruptive. .6,87,92 $^{86}$

That last study retained $93.1 \%$ of participants compared to rates $(64 \%-66 \%)$ seen in the two prior studies enrolling participants new to buprenorphine maintenance treatment (Ling et al and Rosenthal et al). ${ }^{86,87,92}$ Apart from the referral from office-based practices and the required clinical stability of participants who were randomized into the study, other factors that might have contributed to the high overall study retention are that the majority of the participants enrolled were white, employed, had at least a high school education, and were dependent on prescription opioids, rather than heroin. In addition, there may be a contribution to the retention of enhanced therapeutic alliance in participants referred by their psychiatrists or addiction medicine primary care physicians, which might not be as robust for those in an addiction clinic. This suggests potential target populations who share similar characteristics to the study population, such as patients who are dependent on prescription pain medications or who are treated in office-based practices. Characteristics such as a history of cocaine use or having self-identified as white predicted better opioid use outcomes with methadone as compared to traditional treatment with sublingual buprenorphine in a prospective long-term follow-up study, so it would be important to study whether long-acting buprenorphine preparations have an ameliorative impact in those subpopulations. ${ }^{93}$

Since the risk of opioid relapse after cessation of sublingual buprenorphine treatment seems to be inversely associated with the maintenance buprenorphine dosages, ${ }^{94}$ the indication of the implant for those stabilized on a moderatedose sublingual buprenorphine may suggest considering treating those whose daily dose of illicit opioids is relatively lower. In addition, other potential target populations may be those in previously low access populations such as those in the criminal justice system, those being released from incarceration and others. ${ }^{95}$

It is a clinical reality that patients with OUD still tend to sporadically use illicit opioids at different times during maintenance treatment; however, total illicit opioid use during treatment with buprenorphine implants shows a reduction compared to placebo as well as sublingual buprenorphine, substantiating a clinically relevant impact. ${ }^{91,92}$ Over and above the well-demonstrated effects of buprenorphine in the treatment of OUD, what specific aspects of the implant delivery system for buprenorphine are major contributors to its extended clinical utility is as yet unclear. Interestingly, illicit opioid use during maintenance treatment with doses of 
medication effective in blocking the $\mu$-receptor response to agonists may have a predictive value for positive outcomes via extinction processes when patients do not experience reinforcement after opioid use. ${ }^{96}$ One of the potential gains of implants is that they demonstrate their additional effectiveness over time. In the head-to-head double-blind study with sublingual buprenorphine, the buprenorphine implant began to demonstrate a significantly higher responder rate during the third month of treatment, which was then sustained through 6 months. ${ }^{92}$ One hypothesis is that the elimination of daily peak and trough effects after the achievement of steady state reduces the risk of relapse to illicit opioid use. Given that intermediate-range ( 1 week, 30 day) extended release buprenorphine depots are being tested in clinical trials, one should expect to see similar reductions in illicit opioid use against placebo, or perhaps at least at levels intermediate to that seen with daily formulations and the 6-month implant.

\section{Impact of long-acting buprenorphine preparations Adherence risk in high severity opioid users}

Although both methadone and buprenorphine were effective at reducing the use of opioids compared to an untreated population, opioid and heroin use were higher at an average of 4.5 years long-term follow-up in patients randomized to 24-week treatment with sublingual Bup/NTX as compared to oral methadone for OUD, but demonstrated no betweengroups differences in mortality. ${ }^{93}$ The difference in opioid use was attributable to differences in continued participation in treatment with probable differences in medication adherence. ${ }^{93,97}$ In the same study, participants who clustered into a high opioid use trajectory over the follow-up period had significantly fewer months of sublingual buprenorphine treatment than the low-use group, supporting a cumulative medication exposure effect. ${ }^{97}$ Hence, by eliminating the need for frequent dosing and reducing the capacity for patient interaction with the medication, long-acting formulations of buprenorphine may enhance treatment efficacy through improving treatment exposure.

\section{Diversion and illicit self-administration}

Clearly, a medication formulation that is not self-administered, such as a depot or implant, offers a potential advantage in that reducing access to the medicine by patients lowers the risk for diversion. In patients who are non-adherent to the regimen, physical barriers can deter tampering without generating adverse events. ${ }^{98}$ A specific obstacle to diversion is that the buprenorphine implants are under the skin, and once the implantation incision is healed, a minor surgery is required to remove. In addition, the buprenorphine content of the implant is distributed throughout the VPA matrix rather than being deposited in a reservoir state, which makes it more difficult to extract for the purposes of diversion or illicit intravenous use. ${ }^{83}$ Buprenorphine is not quickly separated from the implant matrix by soaking it in water. ${ }^{83}$ However, 4-5 days exposure to an aqueous medium can release $90 \%-95 \%$ of the buprenorphine, and an ethanol or methanol wash for 24 hours will accelerate its extraction in vitro (Sonnie Kim, personal communication). ${ }^{99}$

Similarly, the two aforementioned buprenorphine formulations currently in the development of subcutaneous injection (RBP-6000 and CAM2038) have properties that reduce the likelihood of intravenous use as they either solidify or become a viscous gel, each of which is stationary on contact with bodily fluids. ${ }^{72,77}$ Also, the solid or gel interface that is produced is the rate-limiting step in the diffusion of buprenorphine into bloodstream, so one might expect that the knowledge of this characteristic by patients with OUDs will reduce the likelihood of parenteral application.

\section{Other potential advantages of long-acting buprenorphine preparations}

In clinical circumstances where monitoring or observed dosing of buprenorphine is required, such as in Opioid Treatment Programs in the US, ${ }^{100}$ the reduced frequency of dosing of long-acting forms of buprenorphine may support reduced allocation of clinical staff time to patient monitoring, and thus potentially increase treatment capacity. ${ }^{83}$ Certainly, long-acting preparations lend themselves for use within busy primary care practices, where the availability of physicians with training in addiction medicine and buprenorphine prescribing capacity may be relatively low, but where the clinical staff of other disciplines may be more available to support the ongoing active treatment and overall monitoring of OUDs. However, the need for weekly or monthly dosing is likely to drive regular contact with the clinician. ${ }^{101}$

In particular, the subcutaneous formulations may offer comparable dosing and titration flexibility to sublingual tablets and films, since the dosing can be adjusted based on the volume of injection, but with lower potential for abuse or diversion.

There are high rates of relapse to illicit opioid use after simple medical detoxification. ${ }^{102,103}$ As cumulative exposure to buprenorphine over time reduces relapse rates, ${ }^{97}$ shorter acting weekly or monthly subcutaneous depot formulations 
might be employed for the purposes of extending the period of acute detoxification in addition to titration against symptoms of opioid withdrawal for the purposes of clinical stabilization and maintenance. With the availability of subcutaneous injections, the dosing interval could move from daily sublingual to weekly subcutaneous administration, and the dose might be adjusted clinically as needed. With the patient's continuing clinical stabilization and decreasing impairment, the dosing interval might be moved to once-monthly injections. For those aiming at near-term cessation of medical maintenance, a lower maintenance dose prior to discontinuation may lower the relapse rate. ${ }^{104}$ For those patients for whom longer-term treatment is indicated or desired, monthly subcutaneous injections or 6-month implants may aid in further stabilization. These new delivery systems offer a potential continuum of dosing strength and interval strategies for buprenorphine treatment of OUD.

\section{Summary}

OUDs have continued to cause significant and increasing morbidity and mortality and have reached epidemic proportions in certain regions. Buprenorphine-based maintenance medications have proved to be an important advancement in the effective treatment of OUD, but daily dosing strategies also have associated liabilities such as under-treatment due to missed doses and public health concerns such as purposeful diversion and accidental poisoning, especially in young children. Novel delivery systems for buprenorphine in injectable and implantable forms seem to add a significant advantage through reduction in an individual's physical control of the medication, which increases the probability of consistent treatment exposure over time associated with improved outcomes and reduced opportunity for diversion or accidental exposure.

\section{Disclosure}

RN Rosenthal reports research grants and non-financial support from Braeburn Pharmaceuticals and has served as a consultant for Indivior Inc. and Alkermes, Inc. VV Goradia reports no conflicts of interest in this work.

\section{References}

1. Murray CJL, Vos T, Lozano R, et al. Disability-adjusted life years (DALYs) for 291 diseases and injuries in 21 regions, 1990-2010: a systematic analysis for the Global Burden of Disease Study 2010. Lancet. 2012;380:2197-2223.

2. Rudd RA, Aleshire N, Zibbell JE, Gladden RM. Increases in drug and opioid overdose deaths - United States, 2000-2014. MMWR Morb Mortal Wkly Rep. 2016;64(50):1378-1382.

3. Bahorik AL, Satre DD, Kline-Simon AH, Weisner CM, Campbell CI. Alcohol, cannabis, and opioid use disorders, and disease burden in an integrated health care system. J Addict Med. 2017;11(1):3-9.
4. Hser YI, Mooney LJ, Saxon AJ, et al. High mortality among patients with opioid use disorder in a large healthcare system. J Addict Med. Epub 2017 Apr 20.

5. Rudd RA, Seth P, David F, Scholl L. Increases in drug and opioidinvolved overdose deaths - United States, 2010-2015. MMWR Morb Mortal Wkly Rep. Epub 2016 Dec 16.

6. Dunlap B, Cifu AS. Clinical management of opioid use disorder. JAMA. 2016;316(3):338-339.

7. Substance Abuse and Mental Health Services Administration. Center for Behavioral Health Statistics and Quality. Behavioral Health Trends in the United States: Results from the 2014 National Survey on Drug Use and Health. Washington, DC: Department of Health and Human Services; 2015.

8. Dugosh K, Abraham A, Seymour B, McLoyd K, Chalk M, Festinger D. A systematic review on the use of psychosocial interventions in conjunction with medications for the treatment of opioid addiction. $J$ Addict Med. 2016;10(2):93-103.

9. Weiss RD, Potter JS, Fiellin DA, et al. Adjunctive counseling during brief and extended buprenorphine-naloxone treatment for prescription opioid dependence: a 2-phase randomized controlled trial. Arch Gen Psychiatry. 2011;68(12):1238-1246.

10. Nielsen S, Larance B, Degenhardt L, Gowing L, Kehler C, Lintzeris N. Opioid agonist treatment for pharmaceutical opioid dependent people. Cochrane Database Syst Rev. 2016;5:CD011117.

11. Clark RE, Baxter JD, Aweh G, O'Connell E, Fisher WH, Barton BA. Risk factors for relapse and higher costs among medicaid members with opioid dependence or abuse: opioid agonists, comorbidities, and treatment history. J Subst Abuse Treat. 2015;57:75-80.

12. Amato L, Minozzi S, Davoli M, Vecchi S. Psychosocial combined with agonist maintenance treatments versus agonist maintenance treatments alone for treatment of opioid dependence. Cochrane Database Syst Rev. 2011;10:CD004147.

13. Compton WM, Jones CM, Baldwin GT. Relationship between nonmedical prescription-opioid use and heroin use. N Engl J Med. 2016; 374:154-163.

14. Lofwall MR, Walsh SL. A review of buprenorphine diversion and misuse: the current evidence base and experiences from around the world. J Addict Med. 2014;8(5):315-326.

15. Olfson M, Wang S, Iza M, Crystal S, Blanco C. National trends in the office-based prescription of schedule II opioids. J Clin Psychiatry. 2013;74(9):932-939.

16. Barnett ML, Olenski AR, Jena AB. Opioid-prescribing patterns of emergency physicians and risk of long-term use. N Engl J Med. 2017; 376(7):663-673.

17. Shah A, Hayes CJ, Martin BC. Characteristics of initial prescription episodes and likelihood of long-term opioid use - United States, 2006-2015. MMWR Morb Mortal Wkly Rep. 2017;66:265-269.

18. Kattan JA, Tuazon E, Paone D, et al. Public health detailing - a successful strategy to promote judicious opioid analgesic prescribing. Am J Public Health. 2016;106(8):1430-1438.

19. Dowell D, Zhang K, Noonan RK, Hockenberry JM. Mandatory provider review and pain clinic laws reduce the amounts of opioids prescribed and overdose death rates. Health Aff (Millwood). 2016;35(10): 1876-1883.

20. Passik SD, Hays L, Eisner N, Kirsh KL. Psychiatric and pain characteristics of prescription drug abusers entering drug rehabilitation. $J$ Pain Palliat Care Pharmacother. 2006;20(2):5-13.

21. Iwanicki JL, Severtson SG, McDaniel H, et al. Abuse and diversion of immediate release opioid analgesics as compared to extended release formulations in the United States. PLoS One. 2016;11(12): e0167499.

22. Johnson B, Richert T. Diversion of methadone and buprenorphine by patients in opioid substitution treatment in Sweden: prevalence estimates and risk factors. Int J Drug Policy. 2015;26(2):183-190.

23. Winstock AR, Lea T, Sheridan J. Prevalence of diversion and injection of methadone and buprenorphine among clients receiving opioid treatment at community pharmacies in New South Wales, Australia. Int J Drug Policy. 2008;19:450-458. 
24. Winstock AR, Lea T. Diversion and injection of methadone and buprenorphine among clients in public opioid treatment clinics in New South Wales, Australia. Subst Use Misuse. 2010;45:240-252.

25. Yokell MA, Zaller ND, Green TC, Rich JD. Buprenorphine and buprenorphine/naloxone diversion, misuse, and illicit use: an international review. Curr Drug Abuse Rev. 2011;4:28-41.

26. Johanson CE, Arfken CL, di Menza S, Schuster CR. Diversion and abuse of buprenorphine: findings from national surveys of treatment patients and physicians. Drug Alcohol Depend. 2012;120(1-3):190-195.

27. Fudala PJ, Johnson RE. Development of opioid formulations with limited diversion and abuse potential. Drug Alcohol Depend. 2006; 83(Suppl 1):S40-S47.

28. Larance B, Degenhardt L, Lintzeris N, et al. Post-marketing surveillance of buprenorphine-naloxone in Australia: diversion, injection and adherence with supervised dosing. Drug Alcohol Depend. 2011;118: 265-273.

29. Lintzeris N, Leung SY, Dunlop AJ, et al. A randomised controlled trial of sublingual buprenorphine-naloxone film versus tablets in the management of opioid dependence. Drug Alcohol Depend. 2013;131: 119-126.

30. Lavonas EJ, Severtson SG, Martinez EM, et al. Abuse and diversion of buprenorphine sublingual tablets and film. J Subst Abuse Treat. 2014; 47(1):27-34.

31. Larance B, Lintzeris N, Ali R, et al. The diversion and injection of a buprenorphine-naloxone soluble film formulation. Drug Alcohol Depend. 2014;136:21-27.

32. Robinson SE. Buprenorphine: an analgesic with an expanding role in the treatment of opioid addiction. CNS Drug Rev. 2002;8(4):377-390.

33. Walsh SL, Preston KL, Stitzer ML, Cone EJ, Bigelow GE. Clinical pharmacology of buprenorphine: ceiling effects at high doses. Clin Pharmacol Ther. 1994;55(5):569-580.

34. Walsh SL, Eissenberg T. The clinical pharmacology of buprenorphine: extrapolating from the laboratory to the clinic. Drug Alcohol Depend. 2003;70:S13-S27.

35. Mizoguchi H, Wu HE, Narita M, et al. Antagonistic property of buprenorphine for putative epsilon-opioid receptor-mediated G-protein activation by beta-endorphin in pons/medulla of the mu-opioid receptor knockout mouse. Neuroscience. 2002;115(3):715-721.

36. Mizoguchi H, Spaulding A, Leitermann R, et al. Buprenorphine blocks epsilon- and micro-opioid receptor-mediated antinociception in the mouse. J Pharmacol Exp Ther. 2003;306(1):394-400.

37. Mattick RP, Breen C, Kimber J, Davoli M. Buprenorphine maintenance versus placebo or methadone maintenance for opioid dependence. Cochrane Database Syst Rev. 2014;2:CD002207.

38. Johnson RE, Eissenberg T, Stitzer ML, Strain EC, Liebson IA, Bigelow GE A placebo controlled trial of buprenorphine as a treatment for opioid dependence. Drug Alcohol Depend. 1995;40(1):17-25.

39. Ling W, Charuvastra C, Collins JF, et al. Buprenorphine maintenance treatment of opiate dependence: a multicenter, randomised clinical trial. Addiction. 1998;93(4):475-486.

40. Minozzi S, Amato L, Vecchi S, Davoli M, Kirchmayer U, Verster A. Oral naltrexone maintenance treatment for opioid dependence. Cochrane Database Syst Rev. 2011;2:CD001333.

41. Schottenfeld RS, Chawarski MC, Mazlan M. Maintenance treatment with buprenorphine and naltrexone for heroin dependence in Malaysia: a randomised, double-blind, placebo-controlled trial. Lancet. 2008; 371:2192-2200.

42. Bandawar M, Kandasamy A, Chand P, Murthy P, Benegal V. Adherence to buprenorphine maintenance treatment in opioid dependence syndrome: a case control study. Indian J Psychol Med. 2015;37(3): 330-332.

43. Mokri A, Chawarski MC, Taherinakhost H, Schottenfeld RS. Medical treatments for opioid use disorder in Iran: a randomized, double-blind placebo-controlled comparison of buprenorphine/naloxone and naltrexone maintenance treatment. Addiction. 2016;111(5):874-882.

44. Lopatko OV, White JM, Huber A, Ling W. Opioid effects and opioid withdrawal during a $24 \mathrm{~h}$ dosing interval in patients maintained on buprenorphine. Drug Alcohol Depend. 2003;69:317-322.
45. Kleppner SR, Patel R, McDonough J, Costantini LC. In vitro and in vivo characterization of a buprenorphine delivery system. J Pharm Pharmacol. 2006;58:295-302.

46. Nieuwlaat R, Wilczynski N, Navarro T, et al. Interventions for enhancing medication adherence. Cochrane Database Syst Rev. 2014;11: CD000011.

47. Zweben A, Pettinati HM, Weiss RD, et al. Relationship between medication adherence and treatment outcomes: the COMBINE study. Alcohol Clin Exp Res. 2008;32(9):1661-1669.

48. Casati A, Sedefov R, Pfeiffer-Gerschel T. Misuse of medicines in the European Union: a systematic review of the literature. Eur Addict Res. 2012;18(5):228-245.

49. Boyer EW, McCance-Katz EF, Marcus S. Methadone and buprenorphine toxicity in children. Am J Addict. 2010;19(1):89-95.

50. Martin TC, Rocque MA. Accidental and non-accidental ingestion of methadone and buprenorphine in childhood: a single center experience, 1999-2009. Curr Drug Saf. 2011;6:12-16.

51. Centers for Disease Control and Prevention (CDC). Emergency department visits and hospitalizations for buprenorphine ingestion by children - United States, 2010-2011. MMWR Morb Mortal Wkly Rep. 2013;62(3):56.

52. Budnitz DS, Lovegrove MC, Sapiano MR, et al. Notes from the field: pediatric emergency department visits for buprenorphine/naloxone ingestion - United States, 2008-2015. MMWR Morb Mortal Wkly Rep 2016;65(41):1148-1149.

53. Ritter AJ, Lintzeris N, Clark N, Kutin JJ, Bammer G, Panjari M. A randomized trial comparing levo-alpha acetylmethadol with methadone maintenance for patients in primary care settings in Australia. Addiction. 2003;98(11):1605-1613.

54. Anglin MD, Conner BT, Annon JJ, Longshore D. Longitudinal effects of LAAM and methadone maintenance on heroin addict behavior. J Behav Health Serv Res. 2009;36(2):267-282.

55. Wolstein J, Gastpar M, Finkbeiner T, et al. A randomized, open-label trial comparing methadone and levo-alpha-acetylmethadol (LAAM) in maintenance treatment of opioid addiction. Pharmacopsychiatry. 2009;42(1):1-8

56. Newcombe DA, Bochner F, White JM, Somogyi AA. Evaluation of levo-alpha-acetylmethdol (LAAM) as an alternative treatment for methadone maintenance patients who regularly experience withdrawal: a pharmacokinetic and pharmacodynamic analysis. Drug Alcohol Depend. 2004;76(1):63-72.

57. U.S. Department of Health and Human Services [webpage on the Internet]. Orlaam (levomethadyl acetate hydrochloride). Available from: https://wayback.archive-it.org/7993/20170112171230/http://www.fda. gov/Safety/MedWatch/SafetyInformation/SafetyAlertsforHumanMedicalProducts/ucm153332.htm. Accessed April 4, 2017.

58. Minozzi S, Amato L, Vecchi S, Davoli M, Kirchmayer U, Verster A. Oral naltrexone maintenance treatment for opioid dependence. Cochrane Database Syst Rev. 2006;1:CD001333.

59. Nunes EV, Rothenberg JL, Sullivan MA, et al. Behavioral therapy to augment oral naltrexone for opioid dependence: a ceiling on effectiveness. Am J Drug Alcohol Abuse. 2006;32(4):503-517.

60. Bisaga A, Sullivan MA, Cheng WY, et al. A placebo controlled trial of memantine as an adjunct to oral naltrexone for opioid dependence. Drug Alcohol Depend. 2011;119(1-2):e23-e29.

61. Kunøe N, Lobmaier P, Ngo H, Hulse G. Injectable and implantable sustained release naltrexone in the treatment of opioid addiction. $\mathrm{Br} J$ Clin Pharmacol. 2012;77(2):264-271.

62. Krupitsky E, Nunes EV, Ling W, Illeperuma A, Gastfriend DR, Silverman BL. Injectable extended-release naltrexone for opioid dependence: a double-blind, placebo-controlled, multicentre randomised trial. Lancet. 2011;377(9776):1506-1513.

63. Coviello DM, Cornish JW, Lynch KG, et al. A multisite pilot study of extended-release injectable naltrexone treatment for previously opioiddependent parolees and probationers. Subst Abus. 2012;33(1):48-59.

64. Gordon MS, Kinlock TW, Vocci FJ, et al. A phase 4, pilot, open-label study of VIVITROL ${ }^{\circledR}$ (extended-release naltrexone XR-NTX) for prisoners. J Subst Abuse Treat. 2015;59:52-58. 
65. Lee J, Friedmann P, Kinlock T, et al. Extended-release naltrexone to prevent opioid relapse in criminal justice offenders. $N$ Engl J Med. 2016;374:1232-1242.

66. Hulse GK, Morris N, Arnold-Reed D, Tait RJ. Improving clinical outcomes in treating heroin dependence. Arch Gen Psychiatry. 2009; 66(10):1108-1115.

67. Krupitsky E, Zvartau E, Blokhina E, et al. Randomized trial of longacting sustained-release naltrexone implant vs oral naltrexone or placebo for preventing relapse to opioid dependence. Arch Gen Psychiatry. 2012;69(9):973-981.

68. Das NG, Das SK. Development of mucoadhesive dosage forms of buprenorphine for sublingual drug delivery. Drug Deliv. 2004;11(2): 89-95.

69. Sullivan JG, Webster L. Novel buccal film formulation of buprenorphinenaloxone for the maintenance treatment of opioid dependence: a 12-week conversion study. Clin Ther. 2015;37(5):1064-1075.

70. Sigmon SC, Wong CJ, Nuwayser E, Chausmer AL, Liebson IA, Bigelow GE. Evaluation of an injection depot formulation of buprenorphine. Addiction. 2004;99:1439-1449.

71. Sigmon SC, Moody DE, Nuwayser ES, Bigelow GE. An injection depot formulation of buprenorphine: extended bio-delivery and effects. Addiction. 2006;101(3):420-432.

72. Packhaeuser CB, Schnieders J, Oster CG, Kissel T. In situ forming parenteral drug delivery systems: an overview. Eur J Pharm Biopharm. 2004;58:445-455.

73. Nasser AF, Heidbreder C, Gomeni R, Fudala PJ, Zheng B, Greenwald MK. A population pharmacokinetic and pharmacodynamic modelling approach to support the clinical development of RBP-6000, a new, subcutaneously injectable, long-acting, sustained-release formulation of buprenorphine, for the treatment of opioid dependence. Clin Pharmacokinet. 2014;53(9):813-824.

74. Nasser AF, Greenwald MK, Vince B, et al. Sustained-release buprenorphine (RBP-6000) blocks the effects of opioid challenge with hydromorphone in subjects with opioid use disorder. J Clin Psychopharmacol. 2016;36(1):18-26.

75. Laffont CM, Gomeni R, Heidbreder C, Jones JP 3rd, Nasser AF. Population pharmacokinetic modeling after repeated administrations of RBP-6000, a new, subcutaneously injectable, long-acting, sustainedrelease formulation of buprenorphine, for the treatment of opioid use disorder. J Clin Pharmacol. 2016;56(7):806-815.

76. Indivior [webpage on the Internet]. Indivior announces positive top-line phase 3 pivotal study results for RBP-6000 buprenorphine monthly depot for the treatment of opioid use disorder [press release] [August 17, 2016]. Available from: http://www.indivior.com/investor-news/ rbp-6000-phase-3-top-line-results/. Accessed May 2, 2017.

77. Tiberg F, Johnsson M. Drug delivery applications of non-lamellar liquid crystalline phases and nanoparticles. J Drug Delivery Sci Technol. 2011;21(1):101-109.

78. Tiberg F, Johnsson M, Jankunec M, Barauskas J. Phase behavior, functions, and medical applications of soy phosphatidylcholine and diglyceride lipid compositions. Chem Lett. 2012;41:1090-1092.

79. Braeburn Pharmaceuticals and Camurus announce positive topline phase 3 results for long-acting buprenorphine for treatment of opioid addiction [press release]. CAM-2035 [November 14, 2016]. Available from: http://mb.cision.com/Main/13456/2125166/591383. pdf. Accessed March 4, 2017.

80. Lanier RK, Umbricht A, Harrison JA, Nuwayser ES, Bigelow GE. Evaluation of a transdermal buprenorphine formulation in opioid detoxification. Addiction. 2007;102:1648-1656.

81. Lanier RK, Umbricht A, Harrison JA, Nuwayser ES, Bigelow GE. Opioid detoxification via single 7-day application of a buprenorphine transdermal patch: an open-label evaluation. Psychopharmacology (Berl). 2008; 198:149-158

82. Wiegand TJ, Le Lait MC, Bucher Bartelson B, Dart RC, Green JL. Analysis of the abuse and diversion of buprenorphine transdermal delivery system. J Pain. 2016;17(6):745-752.
83. White J, Bell J, Saunders JB, et al. Open-label dose-finding trial of buprenorphine implants (Probuphine) for treatment of heroin dependence. Drug Alcohol Depend. 2009;103(1-2):37-43.

84. Kuhlman JJ Jr, Levine B, Johnson RE, Fudala PJ, Cone EJ. Relationship of plasma buprenorphine and norbuprenorphine to withdrawal symptoms during dose induction, maintenance and withdrawal from sublingual buprenorphine. Addiction. 1998;93:549-559.

85. Elkader A, Sproule B. Buprenorphine: clinical pharmacokinetics in the treatment of opioid dependence. Clin Pharmacokinet. 2005; 44:661-680.

86. Ling W, Casadonte P, Bigelow G, et al. Buprenorphine implants for treatment of opioid dependence: a randomized controlled trial. JAMA. 2010;304(14):1576-1583.

87. Rosenthal RN, Ling W, Casadonte P, et al. Buprenorphine implants for treatment of opioid dependence: randomized comparison to placebo and sublingual buprenorphine/naloxone. Addiction. 2013; 108(12):2141-2149.

88. O'Connor PG. Buprenorphine implants and opioid dependence - in reply. JAMA. 2011;305:253-255.

89. Basu D, Kumar V. Buprenorphine implants and opioid dependence. JAMA. 2011;305:253-254.

90. Rastegar DA. Buprenorphine implants and opioid dependence. JAMA. 2011;305:253.

91. Ling W, Rosenthal RN, Beebe KL. Buprenorphine implants and opioid dependence. JAMA. 2011;305:254.

92. Rosenthal RN, Lofwall MR, Kim S, et al; PRO-814 Study Group. Effect of buprenorphine implants on illicit opioid use among abstinent adults with opioid dependence treated with sublingual buprenorphine: a randomized clinical trial. JAMA. 2016;316(3): 282-290.

93. Hser YI, Evans E, Huang D, et al. Long-term outcomes after randomization to buprenorphine/naloxone versus methadone in a multi-site trial. Addiction. 2016;111(4):695-705.

94. Bentzley BS, Barth KS, Back SE, Book SW. Discontinuation of buprenorphine maintenance therapy: perspectives and outcomes. J Subst Abuse Treat. 2015;52:48-57.

95. Compton WM, Volkow ND. Improving outcomes for persons with opioid use disorders buprenorphine implants to improve adherence and access to care. JAMA. 2016;316(3):277-279.

96. Mishlen K, Barbieri VA, Maria A, Sullivan MA, Bisaga A. Opioid use following an outpatient detoxification and induction onto XR-NTX: testing the blockade as a predictor of retention in treatment. Drug Alcohol Depend. 2017;171:e146.

97. Hser YI, Huang D, Saxon AJ, et al. Distinctive trajectories of opioid use over an extended follow-up of patients in a multisite trial on buprenorphine+naloxone and methadone. J Addict Med. 2017; 11(1):63-69

98. Stanos SP, Bruckenthal P, Barkin RL. Strategies to reduce the tampering and subsequent abuse of long-acting opioids: potential risks and benefits of formulations with physical or pharmacologic deterrents to tampering. Mayo Clin Proc. 2012;87(7):683-694.

99. Patel RA, Bucalo LR [webpage on the Internet]. Implantable polymeric device for sustained release of buprenorphine. United States patent 7,736,665 United States Patent and Trademark Office. 2010 Jun 15. Available from: http://patft.uspto.gov/netacgi/nph-Parser?S ect $1=$ PTO $1 \&$ Sect $2=$ HITOFF $\& \mathrm{~d}=$ PALL $\& \mathrm{p}=1 \& \mathrm{u}=\% 2$ Fnetahtml $\% 2 \mathrm{~F}$ PTO\%2Fsrchnum.htm\&r=1\&f=G\&l=50\&s1=7,736,665.PN.\&OS=P N/7,736,665\&RS=PN/7,736,665. Accessed April 10, 2017.

100. US Code of Federal Regulations [webpage on the Internet]. 42 CFR §8.12. Available from: https://ecfr.io/Title-42/se42.1.8_112. Accessed February 6, 2017.

101. Remington GJ, Adams ME. Depot neuroleptic therapy: clinical considerations. Can J Psychiatry. 1995;40:S5-S11.

102. Woody GE, Poole SA, Subramanium G, et al. Extended vs short-term buprenorphine-naloxone for treatment of opioid-addicted youth: a randomized trial. JAMA. 2008;300(17):2003-2011. 
103. Bailey GL, Herman DS, Stein MD. Perceived relapse risk and desire for medication assisted treatment among persons seeking inpatient opiate detoxification. J Subst Abuse Treat. 2013;45(3):302-305.
104. Hillhouse M, Canamar CP, Doraimani G, Thomas C, Hasson A, Ling W. Participant characteristics and buprenorphine dose. Am J Drug Alcohol Abuse. 2011;37(5):453-459.

\section{Publish your work in this journal}

Drug Design, Development and Therapy is an international, peerreviewed open-access journal that spans the spectrum of drug design and development through to clinical applications. Clinical outcomes, patient safety, and programs for the development and effective, safe, and sustained use of medicines are the features of the journal, which has also been accepted for indexing on PubMed Central. The manuscript management system is completely online and includes a very quick and fair peer-review system, which is all easy to use. Visit http://www.dovepress.com/testimonials.php to read real quotes from published authors.

Submit your manuscript here: http://www.dovepress.com/drug-design-development-and-therapy-journal 